\title{
Addressable Microlens Array to Improve Dynamic Range of Shack-Hartmann Sensors
}

\author{
Hyuck Choo and Richard S. Muller, Life Fellow, IEEE, Member, ASME
}

\begin{abstract}
In this paper, we have demonstrated an addressable array (5-by-5) of high-quality microlenses suitable for application in a Shack-Hartmann (SH) sensor in a microoptical system. Specific lenses in the array can be addressed using a new selection scheme (that we have designed, built, and tested) in which the mechanical resonant frequencies of individual lens-support carriages are varied. Thus, by changing the frequency of the drive voltage, we require only two electrical connections per row in the lens system to identify the selected lens by its resonating focal image. We show that using this lens-identification method will allow us to improve the dynamic range of $\mathrm{SH}$ sensors by a factor of 12-46 above values reported for conventional SH designs.

[2006-0015]
\end{abstract}

Index Terms-Dynamic range, frequency addressing, microlens, optical microelectromechanical systems (MEMS), Shack-Hartmann (SH) sensor.

\section{NOMENCLATURE}

$A_{\text {comb_overlap }}$ Overlapping area between moving and fixed comb fingers.

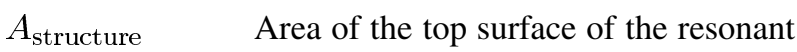
structure.

$A_{\text {structure_overlap }}$ Overlapping area between the resonant structure and substrate.

$b \quad$ Damping factor.

$d_{a} \quad$ Actuation distance.

$d_{\text {lens }}$

Diameter of microlens.

$E_{\mathrm{Si}}$

$f_{\text {lens }}$

Young's modulus of elasticity of silicon.

$f_{r}$

Focal length of the lens.

Resonant frequency.

$g_{c}$

Gap between moving and fixed combs.

$g_{f}$

$k$

Gap between two parallel flexures.

Stiffness or spring constant.

$k_{x-\mathrm{comb}}$

Stiffness or spring constant of the combs in $x$-direction.

$$
\begin{aligned}
& k_{x-\text { flex }} \\
& k_{y-\text { flex }} \\
& k_{y-\max } \\
& l_{c} \\
& l_{f} \\
& l_{\mathrm{ol}}
\end{aligned}
$$

Manuscript received February 7, 2006; revised June 27, 2006. This paper was presented in part at the 2004 Solid-State Sensor, Actuator, and Microsystems Workshop, Hilton Head Island, SCJune6-102004. Subject Editor C. Hierold.

The authors are with the Berkeley Sensor and Actuator Center, Department of Electrical Engineering and Computer Sciences, University of California, Berkeley, Berkeley, CA 94720-1774 USA (e-mail: hchoo@eecs.berkeley.edu).

Color versions of Figs. 3, 8(b), 9, 12, 13, and 15 are available online at http:// ieeexplore.ieee.org.

Digital Object Identifier 10.1109/JMEMS.2006.886011$$
m
$$

$m_{\text {movingstructure }}$

$$
\begin{array}{ll}
m_{\text {flexures }} & \text { Mass of the flexures attached to the unit. } \\
m_{\text {lens }} & \text { Mass of the lens. } \\
n & \text { The number of moving comb fingers. } \\
t_{\text {SOI }} & \text { Thickness of the device layer of the SOI } \\
& \text { wafer. }
\end{array}
$$$$
t_{\mathrm{BOX}}
$$$$
V_{\mathrm{dc}}
$$$$
V_{\mathrm{ac}}
$$$$
V_{\text {dc_dyn }}
$$$$
V_{\text {dc_st }}
$$$$
w_{f}
$$$$
w_{c}
$$$$
y_{0}
$$$$
y_{d}
$$

Stiffness or spring constant of the flexures in $x$-direction.

Stiffness or spring constant of the flexure in $y$-direction.

Maximum stiffness or spring constant of the flexure in $y$-direction allowed in a single row.

Length of combs.

Length of flexures.

Length of overlap between fixed and moving combs.

Total mass of the microlens resonant unit.

Mass of the moving structure without lens and flexures.

Thickness of the buried oxide layer of the SOI wafer.

DC driving voltage.

AC driving voltage.

Maximum dc-driving voltage before side thrust occurs (at resonance).

Maximum dc-voltage before a side thrust occurs (at stationary position).

Width of flexures.

Width of combs.

Initial overlap length between the fixed and moving combs.

Desired actuation distance at resonance in $y$-direction.

$y_{\max \_ \text {is }} \quad$ Maximum actuation distance before a side thrust occurs (at resonance) in $y$-direction.

$y_{r} \quad$ Actuation distance at resonance in $y$-direction.

Permittivity of air. 


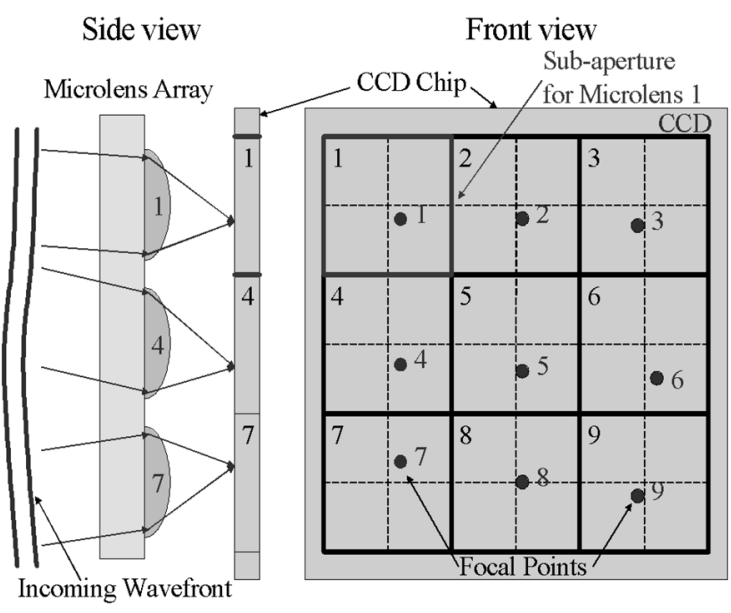

(a)

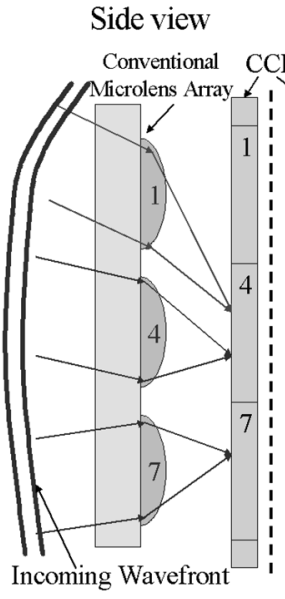

Incoming Wavefront
Front view

Dislocated Focal Spots:

Erroneous Measurements

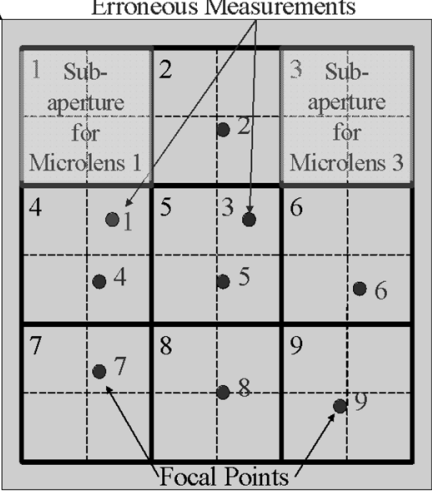

(b)

Fig. 1. (a) Wavefront-slope measurement using microlens array: each microlens has its own subaperture consisting of approximately 40 CCD pixels (divided into four quadrants), and the focal point of the microlens must be located within the assigned subapertures; (b) Limited dynamic range of a conventional Shack-Hartmann sensor (left): a highly aberrated wavefront causes the focal points of microlenses \#1 and \#3 to become focused onto the subapertures assigned to microlenses \#4 and \#5, respectively, causing erroneous measurements.

$\begin{array}{ll}\rho_{\mathrm{Si}} & \text { Density of single crystal silicon. } \\ \rho_{\text {Lens }} & \text { Density of lens material. } \\ \omega & \text { Angular frequency }(2 \cdot \pi \cdot f) . \\ \omega_{\mathrm{r}} & \text { Angular resonant frequency }\left(2 \cdot \pi \cdot f_{r}\right) . \\ \mu & \text { Viscosity of air. } \\ \nu & \text { Kinetic viscosity of air. }\end{array}$

\section{INTRODUCTION}

$\mathbf{S}$ HACK-Hartmann (SH) sensors are widely used in astronomical telescopes and ophthalmic-analysis systems as monitors for wavefront aberrations. They are fast, accurate, and, in contrast to interferometers, generally insensitive to vibrations. When they are used in conjunction with adaptive mirrors, Shack-Hartmann sensors are able to improve the image quality of astronomical telescopes by performing real-time corrections on the wavefront aberrations that are inherently generated as starlight traverses the earth's atmosphere [1]. Shack-Hartmann sensors have also proven to be the most suitable wavefront monitors for ophthalmic-analysis applications (such as preand/or post-LASIK surgery and keratoconus analysis) because measuring the optical aberrations in illumination passing through constantly moving human eyes requires fast measurement speed and high accuracy [2]-[6].

In Shack-Hartmann systems, a microlens array dissects an incoming wavefront into a number of segments [Fig. 1(a)] [7]. Each microlens in the array creates a focal spot within the assigned subaperture on the charge-coupled device (CCD) (typically made of $40 \mathrm{CCD}$ pixels). Because light travels in a straight path normal to the wavefront, the positions of these focal spots are related to the average wavefront slope over each microlens aperture. Thus the pattern of spots at the focal plane contains information about the spatially resolved waveform slope that can be integrated to reconstruct the wavefront. The dynamic range (the range of measurable wavefront slope) of a conventional SH system has fundamental design limits that affect its performance; an SH system produces false results if the curvature of the wavefront being measured is too large [2]. Fig. 1(b) shows one of these cases in which a focal point of one microlens, as a result of extreme aberration in the incoming wave, moves into an adjacent subaperture that has been preassigned to register the focal point of another microlens.

Researchers have attempted to overcome this dynamic-range limitation of SH systems using at least three methods: 1) by employing a modified unwrapped algorithm [8], 2) by using an SH array of microlenses with well-defined astigmatism [9], or 3) by positioning a spatial-light modulator in front of the $\mathrm{SH}$ microlens array as a shutter [10]. Research showed that the first two methods had limited practical use providing accurate measure of wavefront aberration. Method 1) does not work with wavefronts that exhibit localized aberrations of large magnitudes. Method 2 ) requires that the elliptical focal spots have enough space between them along the major and minor axes in order to obtain proper measurements. Hence, the spatial density of the astigmatic microlens array has to be much lower than that of a circular microlens array, and this in turn lowers the accuracy of the sensor. Method 3), which employs a spatial-light modulator, is also impractical on three grounds: the modulator absorbs a great deal of light (at least 50\% in the case of a liquid crystal display illuminated with unpolarized light); it increases the noise in the measurement; and it introduces additional aberrations to the wavefront being measured. In addition, spatial-light modulators can have polarization dependences, and these modulators are typically very expensive.

Nonetheless, expanding the dynamic range of Shack-Hartmann sensors is highly desired, especially in consideration of their increasing uses in refractive surgery $(\$ 600$ million market in 2001) and in keratoconus analysis. In the case of refractive surgery, the development of a transition zone (resultant from scar tissues) at the boundary separating surgically treated and untreated areas results in large optical aberrations [11] when the 


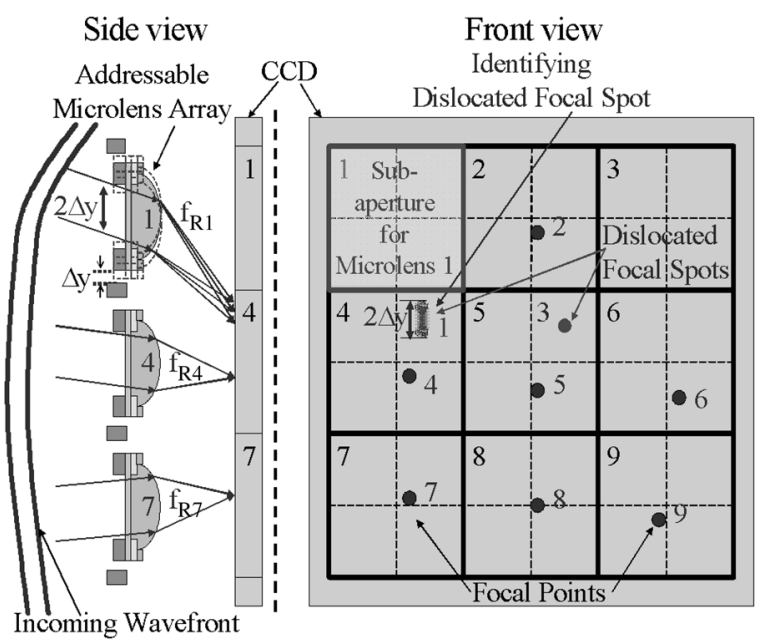

Fig. 2. By making each microlens resonate individually, we can identify its associated focal point, even if the focal point is located outside the assigned subaperture.

tissue is examined. Also analyzing ophthalmic diseases such as keratoconus (meaning cone-shaped cornea) requires large dynamic ranges and sensitivities that cannot be achieved by conventional Shack-Hartmann sensors [2].

Using microelectromechanical systems (MEMS) technologies developed in the Berkeley Microlab, we have created densely packed active microlens arrays in which each of the lenses is designed so that it can be driven to resonate at a predesigned frequency. When a lens resonates, its focal point moves parallel to its motional direction [12], [13]; hence by selecting the frequency of the driving voltage on a string of parallel-connected lenses, we can select only the lens that is resonant at the driving frequency. We can then identify the focal point of that resonating lens by detecting a line instead of a point image Fig. 2. To build the system, we have designed the individual lens carriages for the array of lenses to have separated natural resonant frequencies so that, by changing the frequency of the drive voltage, we require only two electrical connections per lens-carriage row to identify the selected lens.

With our design, a lens focal point can be identified even when it falls outside of its associated subaperture (usually 40 pixels for Shack-Hartmann sensors for ophthalmic analysis), anywhere in the sensing array. This identification will allow the dynamic range of the Shack-Hartmann sensor to be dramatically improved-a factor of 26 to 46 better than that achieved in conventional Shack-Hartmann systems. In this paper, we discuss the design, fabrication, and experimental results on our addressable microlens array.

\section{AdDRESSABle Microlens-ARRAY DESIGN}

\section{A. Design Objectives and Considerations for Addressable Microlens Array}

The design of our addressable microlens array for Shack-Hartmann sensors has been guided by the following objectives.
1) Maximize the clear aperture of the system (or microlens area) by minimizing the areas for MEMS actuators and electrical interconnects.

2) Assure that only the desired lens moves appreciably while all other lenses in its row remain essentially stationary, even when the lens carriage with the stiffest flexures (highest resonant frequency) in the row is actuated with the highest drive voltage.

The first objective is reached by designing and building the lens-carriages using the most efficient surface-micromachining capabilities available in our laboratory. Achieving the second objective requires the simultaneous consideration of the frequency responses of all the units in a single row and the analysis of side instabilities of the resonating units. The side-instabilities are caused by the electrostatic pull-in phenomena [14], which we discuss in more detail later in this paper. The frequency responses and side-thrust issues will determine the maximum number of the MEMS-microlens units per row that can be reliably addressed by our frequency-addressing method.

\section{B. Layout and Dimensions of the Addressable-Microlens Array}

Fig. 3 shows an enlarged view of an individual MEMS-microlens unit and the schematic diagram of our addressable-microlens array.

In order to utilize the area inside the unit rectangular cell (1360 by $1460 \mu \mathrm{m}^{2}$ ) efficiently, the microlens is placed at the center of the cell, the two truss-joined double flexures are placed at the top and bottom of the cell, and comb-fingers (numbering 172 moving and 184 fixed) are concentrated between the two flexures, around the microlens [Fig. 3(a)], inside the cell. The comb-fingers are grouped into upper and lower comb sets, as shown in Fig. 3(a). The upper comb sets are mirror images of the lower comb sets reflected across the $x$-axis. The truss-joined double flexures have been chosen to maximize pliability along the actuation directions of the resonant structures as well as to stiffen their resistances to undesired sideway motions.

Each row contains five adjacent microlens-resonant units designed to have five different mechanical-resonant frequencies. The frequencies are varied by decreasing the support-flexure lengths from 900 to $500 \mu \mathrm{m}$ in steps of $100 \mu \mathrm{m}$, from the left (Unit 1) to the right (Unit 5) [Fig. 3(b)]. Using our frequency-addressing method, we need only a single pair of interconnects per row to select and energize each unit, reducing and simplifying the area and complexity necessary for alternative selection designs. A 5-by-5 addressable array is then obtained by stacking five identical rows (Row1-Row5), as shown in Fig. 3(b). The dimensions of the MEMS-microlens resonant structures are summarized in Table I, while other relevant material parameters are listed in Table II. The reasons for choosing the listed values for the dimensions will be clarified in the following sections.

\section{Design of MEMS Resonators With Electrostatic Actuators}

In order to assure the successful, distinctive resonant motion of each MEMS-microlens unit, the following requirements must be met. (Please refer to Fig. 4.) First, each unit must be able to achieve $\pm 20-\mu \mathrm{m}$ amplitude at resonance stably and without appreciable sideway motions. Secondly, when the unit 


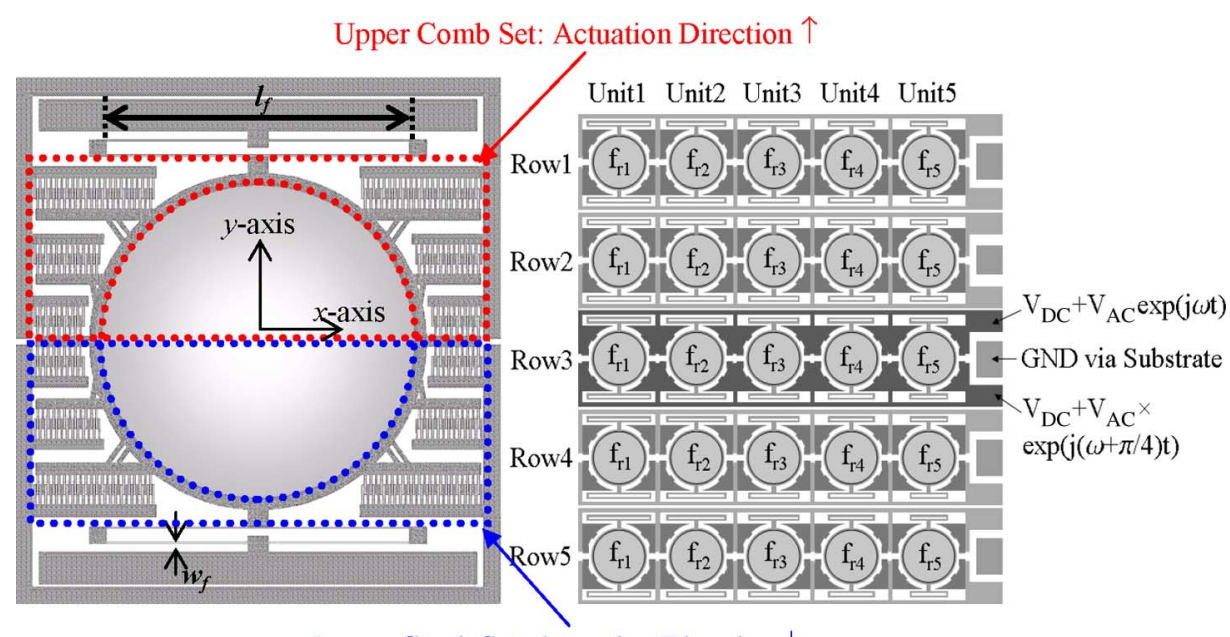

Lower Comb Set: Actuation Direction $\downarrow$

(a)

(b)

Fig. 3. Schematic diagrams showing features of addressable-microlens array. (a) Individual resonant unit. (b) Our resonant-frequency addressing method requires only a single pair of electrical lines per row to control each unit individually. Rows 1-5 are identical; each row contains five MEMS-microlens units having five different resonant frequencies $\left(\mathrm{f}_{\mathrm{r} 1}-\mathrm{f}_{\mathrm{r} 5}\right)$.

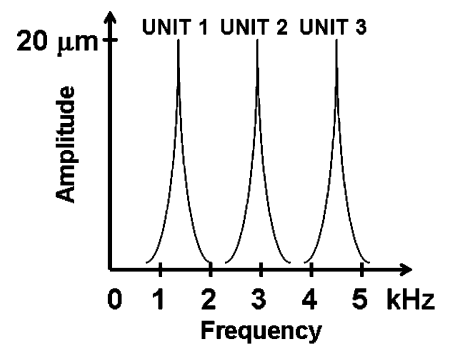

(a)

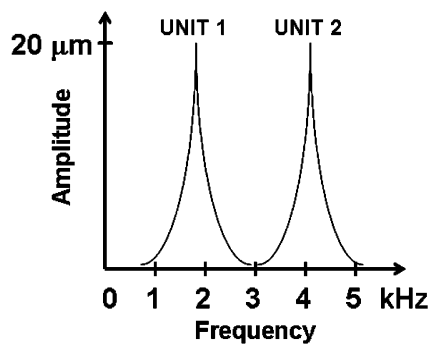

(b)

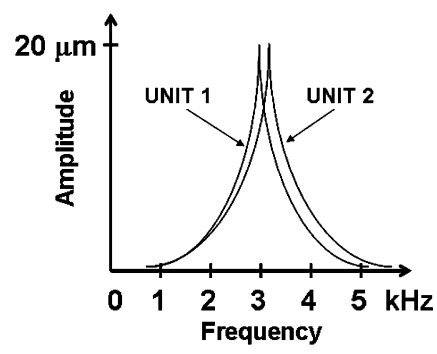

(c)

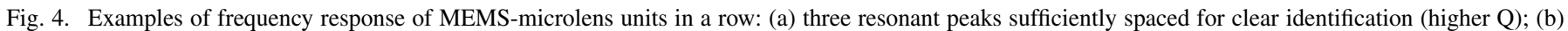

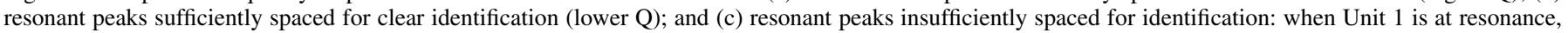
Unit 2 will also show considerable movements, making it difficult to identify the units.

TABLE I

RELEVANT PARAMETERS OF THE MEMS-MICROLENS UNITS

\begin{tabular}{|l|c|}
\hline Number of moving comb-fingers per unit & 172 \\
\hline Length $\left(\boldsymbol{I}_{\boldsymbol{c}}\right)$ and width $\left(\boldsymbol{w}_{c}\right)$ of comb-fingers $(\mu \mathrm{m})$ & 60,5 \\
\hline Length $\left(\boldsymbol{l}_{f}\right)$ and width $\left(\boldsymbol{w}_{f}\right)$ of flexures $(\mu \mathrm{m})$ & $500-900,4$ \\
\hline Gap between comb-fingers $\left(g_{c}\right)(\mu \mathrm{m})$ & 3 \\
\hline Thickness of SOI-wafer device layer $\left(t_{S O I}\right)(\mu \mathrm{m})$ & 20 \\
\hline Thickness of SOI-wafer buried-oxide-layer $\left(t_{B O X}\right)(\mu \mathrm{m})$ & 2 \\
\hline Diameter of integrated microlens $\left(d_{\text {lens }}\right)(\mu \mathrm{m})$ & 800 \\
\hline Focal length of microlens $\left(f_{\text {lens }}\right)(\mathrm{mm})$ & 2 \\
\hline
\end{tabular}

TABLE II

RELEVANT MATERIAL PARAMETERS FOR THE MEMS-MiCROLENS UNITS

\begin{tabular}{|l|c|}
\hline Young's Modulus $\left(E_{S i}\right)$ of silicon $(\mathrm{GPa})$ & 170 \\
\hline Density of silicon $\left(\rho_{\mathrm{S} i}\right)\left(\mathrm{kg} / \mathrm{m}^{3}\right)$ & 2330 \\
\hline Density of microlens material $\left(\rho_{\text {lens }}\right)\left(\mathrm{g} / \mathrm{cm}^{3}\right)$ & 1.08 \\
\hline Refractive index of microlens material $(n)$ & 1.51 \\
\hline Viscosity of air $(\mu)\left(\mathrm{N} \cdot \mathrm{s} / \mathrm{m}^{2}\right)$ & $1.8 \cdot 10^{-5}$ \\
\hline Kinetic viscosity of air $(\mathrm{v})\left(\mathrm{m}^{2} / \mathrm{s}\right)$ & $1.5 \cdot 10^{-5}$ \\
\hline Permittivity of air $(\varepsilon)(\mathrm{F} / \mathrm{m})$ & $8.854 \cdot 10^{-12}$ \\
\hline
\end{tabular}

with the highest resonant frequency (which has the stiffest flexures and therefore requires the highest driving voltage) achieves $\pm 20-\mu \mathrm{m}$ resonant amplitude, all other units must move only negligibly and be free from any undesired side thrust. Thirdly, the resonant peak of each MEMS-microlens unit should be sufficiently separated in frequency from the resonant peaks of the other units in the same row.

A side-thrust mentioned in the first and the second requirements is caused by the electrostatic pull-in phenomenon [14], which is illustrated in Fig. 5. The moving combs are sometimes not perfectly centered between the fixed combs due to the processing variations Fig. 5(c). And, even if the combs were initially aligned perfectly, they can slightly deviate from the ideal line of actuation when the structures are actuated or some external vibrations are present. This slight misalignment results in an unbalanced net electrostatic force in the direction ( $x$-axis) perpendicular to the desired actuation ( $y$-axis) [Fig. 5(c)]. When the changes in these electrostatic forces with respect to $x$ become larger than the stiffness of the flexures along the $x$-axis, the moving comb-fingers will bend and in all likelihood stick to the fixed combs, causing the proper operation of the structure to fail, as shown in Fig. 5(d). We call the voltage at which the structure begins to fail in this way the side-thrust voltage.

There are two different cases of side thrust that we consider. The first case, which we call dynamic side thrust, may occur 


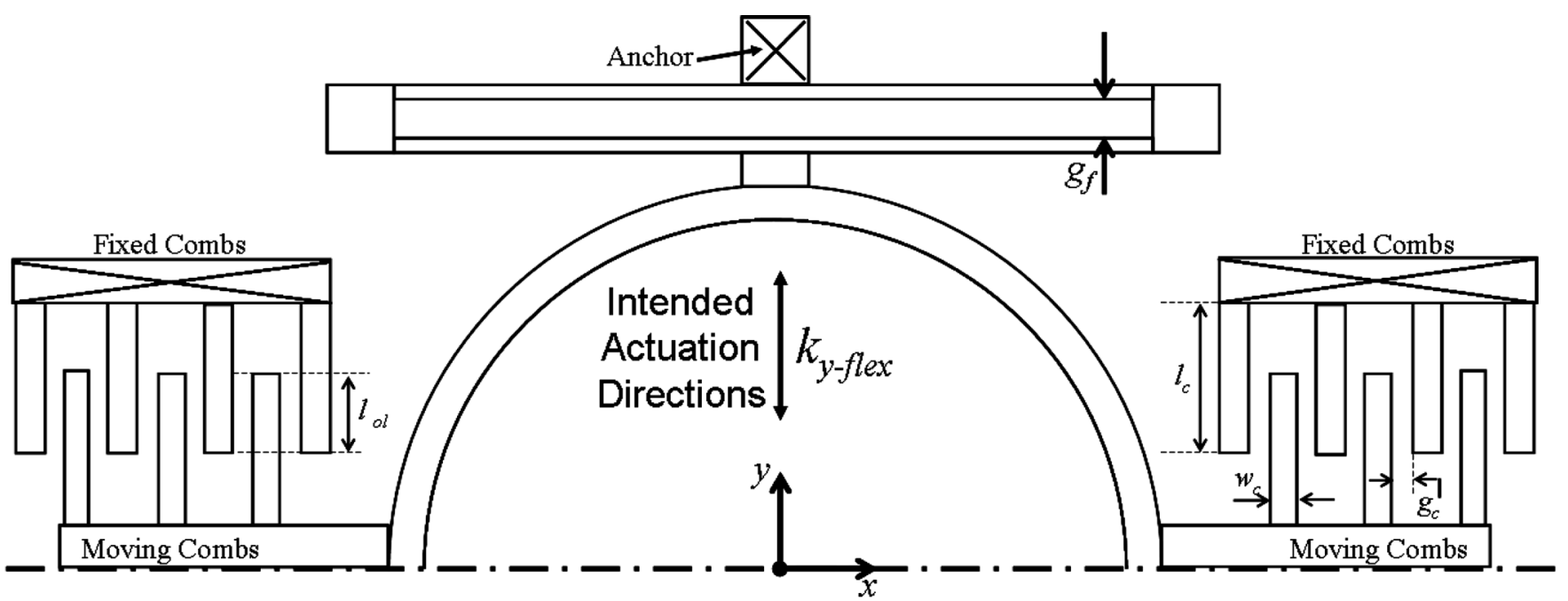

(a)

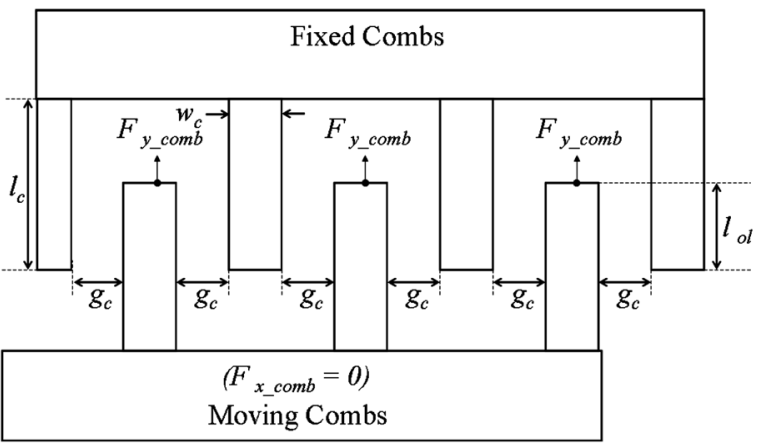

(b)

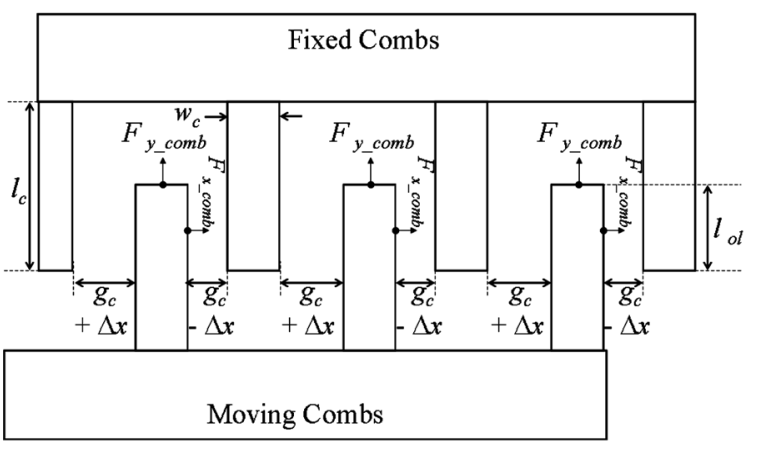

(c)

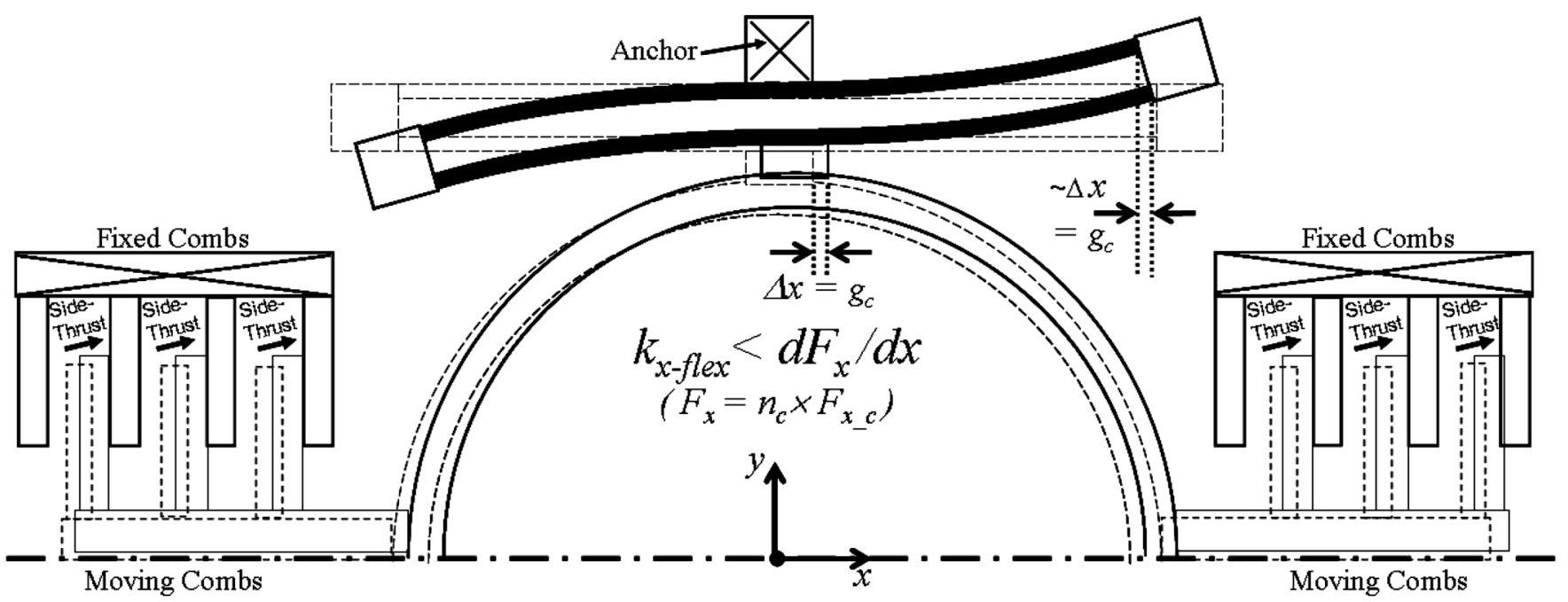

(d)

Fig. 5. (a) Sketches of the upper half of a MEMS-microlens unit; (b) perfectly aligned fixed and moving combs; (c) misaligned fixed and moving combs-the gaps on the right side of the moving combs are smaller than those the gap on the left side of the moving combs, causing unbalanced electrostatic force in the x-direction. (d) The force $F_{x}$ generated by the misaligned combs causes the unit to shift to the left by $\Delta x$. If the change in $F_{x}$ is larger than the stiffness of the flexures in $x$-direction, $\Delta x$ becomes as large as $g_{c}$, and the moving-comb fingers become sufficiently displaced to cause electrical contact with the fixed-comb fingers, shorting out the drive voltage.

to the unit at resonance when its drive voltage is increased to achieve the intended resonant amplitude. The second case, stationary side thrust, occurs when the unit with the highest resonant frequency is driven to the desired resonant amplitude while the others are stationary. We need to consider them separately because the overlapping areas between the moving- and fixed-comb fingers remain constant for stationary side thrust yet change in the case of dynamic side thrust. For stationary side thrust, some of the other lower resonant-frequency units may experience side thrust because the highest frequency unit has the stiffest flexures and requires the highest drive voltage. This high drive voltage is applied to all the units in the row, possibly causing one or more of them to move in an undesired direction and to fail. 
TABLE III

PREDicted Resonant FreQuencies AND Quality Factors of THE Five MEMS-MICROLENS RESONANT UNITS IN A ROW

\begin{tabular}{|l|l|l|l|l|l|}
\hline & Unit1 & Unit2 & Unit3 & Unit4 & Unit5 \\
\hline $\boldsymbol{l}_{\boldsymbol{f}}(\boldsymbol{\mu \mathrm { m }})$ & 900 & 800 & 700 & 600 & 500 \\
\hline $\boldsymbol{w}_{\boldsymbol{f}}(\boldsymbol{\mu \mathrm { m } )})$ & 4 & 4 & 4 & 4 & 4 \\
\hline $\boldsymbol{f}_{\boldsymbol{r}}(\boldsymbol{k H z})$ & 1.56 & 1.88 & 2.33 & 3.00 & 4.05 \\
\hline $\boldsymbol{Q}$ & 116.13 & 139.67 & 172.44 & 220.43 & 295.80 \\
\hline
\end{tabular}

Considering both types of failures and the needed selectivity among the lenses in a given row, we see that the number of units in a row is determined by (a) the upper and lower limits on resonant frequencies set by the side-thrust issues and (b) the quality factors of the resonant units in the row, which determine the spacing needed between adjacent units in the frequency domain for clear distinction.

To analyze the effects of these limitations, we begin by formulating expressions for the resonant frequencies, quality factors, and resonant amplitudes of the lens units. Then we consider the range of resonant frequencies allowed in a single row as set by the physical dimensions of the unit cell as well as by the side-thrust issues.

1) Calculations of Resonant Frequencies, Quality Factors, and Resonant Amplitudes: The resonant frequency of each MEMS-microlens unit is [15]

$$
f_{r}=\frac{1}{2 \cdot \pi} \sqrt{\frac{k}{m}}
$$

where $m$ is [16]

$$
m=m_{\text {movingstructure }}+0.3714 \cdot m_{\text {flexures }}+m_{\text {lens }} .
$$

The stiffness $k$ of the flexures is calculated using rectangular beam theory [17]

$$
k_{y-\mathrm{flex}}=\frac{16 \cdot t_{\mathrm{SOI}} \cdot w_{f}^{3} \cdot E_{\mathrm{Si}}}{l_{f}^{3}} .
$$

From (1) and (3), we see that increasing the stiffness $k$ by reducing the flexure length $l_{f}$ increases the resonant frequency $f_{r}$. The mechanical quality factor $Q$ for the resonant carriages is

$$
Q=\frac{\sqrt{k \cdot m}}{b} .
$$

The damping factor $b$ is calculated by summing i) Couette-flow damping between the fixed and moving comb-fingers, ii) Couette-flow damping between the resonant structure and the substrate, and iii) Stokes-flow damping over the resonant structure [18]

$$
\begin{aligned}
& b=\frac{\mu \cdot A_{\text {comb_overlap }}}{g_{c}}+\frac{\mu \cdot A_{\text {structure_overlap }}}{t_{\text {BOX }}}+\frac{\mu \cdot A_{\text {structure }}}{\delta} \\
& \delta=\sqrt{\frac{2 \cdot \nu}{\omega_{r}}} .
\end{aligned}
$$

Using the fundamental resonant frequencies and the $\mathrm{Q}$ factors, we predict the frequency response of the microlens reso nant structures in a row as [15]

$$
y_{\mathrm{ac}}(\omega)=\frac{n_{c} \cdot \varepsilon \cdot t_{\mathrm{SOI}} \cdot\left(V_{\mathrm{dc}}+V_{\mathrm{ac}}\right)^{2}}{g_{c} \cdot\left[\left(k-m \cdot \omega^{2}\right)^{2}+\left(\frac{\omega \cdot \omega_{r} \cdot m}{Q}\right)^{2}\right]^{\frac{1}{2}}} .
$$

In (6), $\omega$ is the angular frequency of the drive voltage and $\omega_{r}$ is the angular resonant frequency of the unit under consideration. As shown in Fig. 3, each MEMS-microlens unit has two sets of comb fingers: an upper and a lower set. The upper and lower sets actuate the MEMS-microlens unit upward and downward, respectively. Each of these sets has an equal number of paired fixed- and moving-comb fingers. Hence, dc voltage simultaneously applied to both upper and lower comb sets causes balanced forces and no displacement; only an ac voltage, that is applied to the upper and lower comb sets with a $90^{\circ}$-phase lag one from the other, will cause displacement. In this case, the equation for the resonant displacement becomes

$$
y_{\mathrm{ac}}(\omega)=\frac{n_{c} \cdot\left(2 \cdot V_{\mathrm{dc}} \cdot V_{\mathrm{ac}}+V_{\mathrm{ac}}^{2}\right)^{2} \cdot \varepsilon \cdot t_{\mathrm{SOI}}}{g_{c} \cdot\left[\left(k-m \cdot \omega^{2}\right)^{2}+\left(\frac{\omega \cdot \omega_{r} \cdot m}{Q}\right)^{2}\right]^{\frac{1}{2}}} .
$$

For cases in which $V_{\mathrm{dc}}$ is much larger than $V_{\mathrm{ac}}, y_{\mathrm{ac}}(\omega)$ can be further simplified as

$$
y_{\mathrm{ac}}(\omega) \approx \frac{n_{c} \cdot\left(2 \cdot V_{\mathrm{dc}} \cdot V_{\mathrm{ac}}\right) \cdot \varepsilon \cdot t_{\mathrm{SOI}}}{g_{c} \cdot\left[\left(k-m \cdot \omega^{2}\right)^{2}+\left(\frac{\omega \cdot \omega_{r} \cdot m}{Q}\right)^{2}\right]^{\frac{1}{2}}} .
$$

The designer must consider (6-1) when it is applied over the frequency range of interest in order to assure that each unit in a given row achieves the desired amplitude at its resonant frequency while movements in the other units in the row are negligible at that frequency. The selected unit must also resonate at the desired amplitude without suffering from excessive dynamic side thrust. At resonance, the amplitude is

$$
y_{r}=y_{\mathrm{ac}}\left(\omega_{r}\right)=\frac{n_{c} \cdot Q \cdot \varepsilon \cdot t_{\mathrm{SOI}}}{k_{y_{-} \text {flex }} \cdot g_{\mathrm{comb}}}\left(V_{\mathrm{dc}}+V_{\mathrm{ac}}\right)^{2} .
$$

For cases in which $V_{\mathrm{dc}}$ is much larger than $V_{\mathrm{ac}}$, the expression for the maximum amplitude at the resonant frequency can be approximated as

$$
y_{r} \approx \frac{n_{c} \cdot Q \cdot \varepsilon \cdot t_{\mathrm{SOI}}}{k_{y_{-} f l e x} \cdot g_{\mathrm{comb}}}\left(2 \cdot V_{\mathrm{dc}} \cdot\left|V_{\mathrm{ac}}\right|\right) .
$$

Turning now to the dynamic side thrust issue, we calculate the maximum attractive electrostatic force between the moving and fixed combs that causes side-thrust at the resonant frequency [14]

$$
\begin{aligned}
F_{\text {Side }}= & \frac{\left(n_{c} / 2\right) \cdot t_{\mathrm{SOI}} \cdot\left(y_{0}+y_{r}\right)}{2\left(g_{c}-x\right)^{2}}\left(V_{\mathrm{dc}}+\left|V_{\mathrm{ac}}\right|\right)^{2} \\
& -\frac{\left(n_{c} / 2\right) \cdot t_{\mathrm{SOI}} \cdot\left(y_{0}+y_{r}\right)}{2\left(g_{c}+x\right)^{2}}\left(V_{\mathrm{dc}}+\left|V_{\mathrm{ac}}\right|\right)^{2} \\
& +\frac{\left(n_{c} / 2\right) \cdot t_{\mathrm{SOI}} \cdot\left(y_{0}-y_{r}\right)}{2\left(g_{c}-x\right)^{2}}\left(V_{\mathrm{dc}}\right)^{2} \\
& -\frac{\left(n_{c} / 2\right) \cdot t_{\mathrm{SOI}} \cdot\left(y_{0}-y_{r}\right)}{2\left(g_{c}+x\right)^{2}}\left(V_{\mathrm{dc}}\right)^{2} .
\end{aligned}
$$




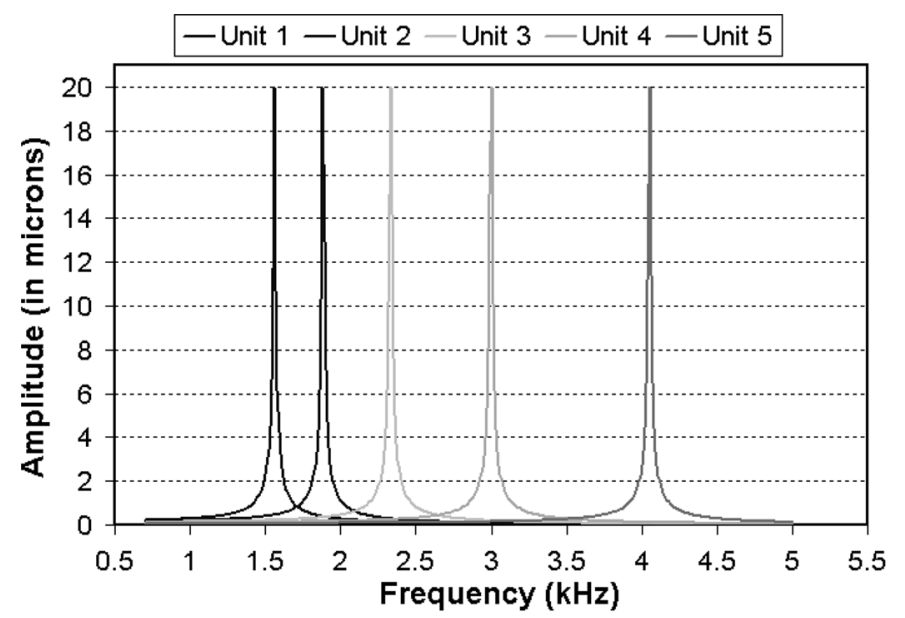

(a)

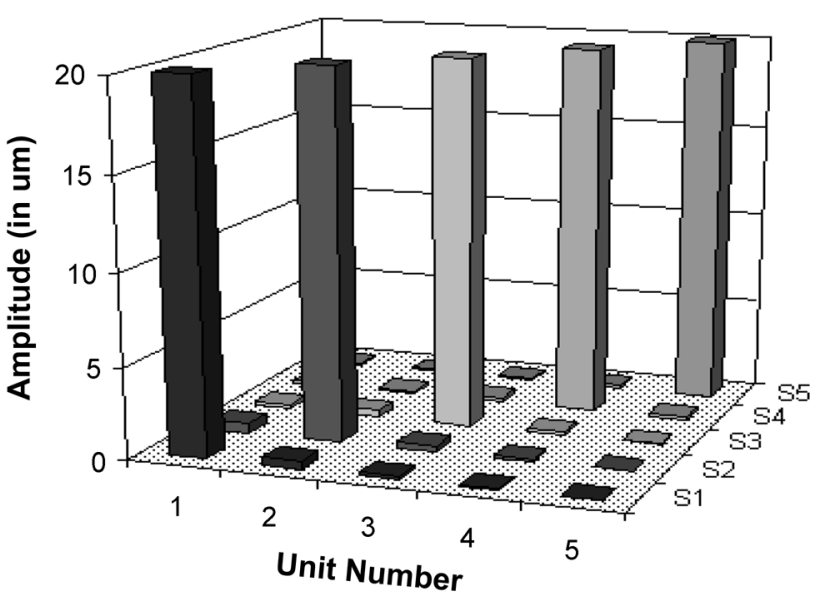

(b)

Fig. 6. (a) Predicted frequency responses of the MEMS-microlens units and (b) graph representing the data in Table IV to emphasize the selectivity of the mechanical-resonance method.

TABLE IV

PREDICTED AMPLITUDES FOR THE MEMS-MICROLENS UNITS AT DIFFERING DRIVE FREQUENCIES: BOLD CHARACTERS INDICATE THE RESONANT UNIT. (A: Amplitude)

\begin{tabular}{|c|c|c|c|c|c|}
\hline & Unit1 & Unit2 & Unit3 & Unit4 & Unit5 \\
\hline$l_{f}(\mu \mathrm{m})$ & 900 & 800 & 700 & 600 & 500 \\
\hline $\begin{array}{c}A \text { (in } \mu \mathrm{m})(\mathrm{S} \text { S: Unit } 1 \text { Designated) } \\
\left(\mathrm{V}_{\text {Applied }}=20 \mathrm{~V}_{\mathrm{ac}}, f_{\text {Applied }}=f_{r_{u}} u n i t I\right)\end{array}$ & 20.45 & 0.44 & 0.17 & 0.08 & 0.04 \\
\hline $\begin{array}{c}A \text { (in } \mu \mathrm{m})(\mathrm{S} 2: \text { Unit } 2 \text { Designated }) \\
\left(\mathrm{V}_{\text {Applied }}=25 \mathrm{~V}_{\mathrm{ac}}, f_{\text {Applied }}=f_{r_{-} \text {unit } 2}\right)\end{array}$ & 0.52 & 20.25 & 0.32 & 0.11 & 0.05 \\
\hline $\begin{array}{c}A(\text { in } \mu \mathrm{m})(\mathrm{S3} \text { : Unit } 3 \text { Designated) } \\
\left(\mathrm{V}_{\text {Applied }}=32 \mathrm{~V}_{\mathrm{ac}}, f_{\text {Applied }}=f_{r_{-} u n i t}\right)\end{array}$ & 0.24 & 0.39 & 20.40 & 0.22 & 0.07 \\
\hline $\begin{array}{c}A \text { (in } \mu \mathrm{m})(\mathrm{S} 4: \text { Unit } 4 \text { Designated) } \\
\left(\mathrm{V}_{\text {Applied }}=42 \mathrm{~V}_{\mathrm{ac}}, f_{\text {Applied }}=f_{r_{-} u n i t}\right) \\
\end{array}$ & 0.14 & 0.17 & 0.27 & 20.23 & 0.14 \\
\hline $\begin{array}{c}A \text { (in } \mu \mathrm{m})(\mathrm{S} 5: \text { Unit } 5 \text { Designated) } \\
\left(\mathrm{V}_{\text {Applied }}=53 \mathrm{~V}_{\mathrm{ac}}, f_{\text {Applied }}=f_{r_{-} u n i t}\right)\end{array}$ & 0.09 & 0.10 & 0.12 & 0.18 & 20.01 \\
\hline
\end{tabular}

In (8), the number of comb fingers $\left(n_{c}\right)$ is divided by two because $V_{\mathrm{ac}}$ is sinusoidal and its peak voltage is applied either to the upper or to the lower comb each half-cycle. The stable operation of the resonant unit requires that

$$
\begin{aligned}
k_{x_{-} \text {flex }} \gg & {\left[\frac{\partial F_{\text {side }}}{\partial x}\right]_{x \rightarrow 0}=\left(\frac{n_{c} \cdot t_{\text {SOI }}}{g_{c}^{3}}\right) } \\
& \times\left[\left(y_{0}+y_{r}\right)\left(V_{\mathrm{dc}}+\left|V_{\mathrm{ac}}\right|\right)^{2}+\left(y_{0}-y_{r}\right) V_{\mathrm{dc}}^{2}\right] .
\end{aligned}
$$

In (9), $k_{x_{-} \text {flex }}$ is the effective stiffness of the truss-joined doubleflexures in the $x$-direction; this parameter can be calculated by considering the slope developed at the end of the flexure beams when stiction takes place [17]

$$
k_{x-\text { flex }}=\frac{4 \cdot t_{\mathrm{SOI}} \cdot E_{\mathrm{Si}} \cdot w_{f}^{3}}{3 \cdot l_{f} \cdot g_{f}^{2}} .
$$

When the drive voltage exceeds the side-thrust voltage $\left(V_{s_{-} d}=\right.$ $\left.V_{\text {dc_dyn }}+\left|V_{\text {ac }}\right|\right)$, the comb drive becomes unstable, leading the moving comb finger to stick to its fixed counterpart. If we express $y_{r}$ using (7-1) in (9) and assume a reasonable value for $\left|V_{\mathrm{ac}}\right|$ (for example, $V_{\mathrm{dc}} \gg\left|V_{\mathrm{ac}}\right|,\left|V_{\mathrm{ac}}\right|=4 \mathrm{~V}$ ), we can solve for $V_{\text {dc_dyn }}$

$$
V_{\mathrm{dc} \_ \text {dyn }}=\frac{-B+\sqrt{B^{2}-4 \cdot A \cdot C}}{2 \cdot A}
$$

where

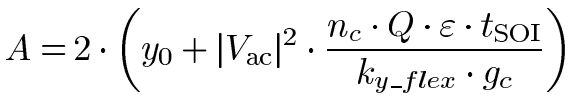

$$
\begin{aligned}
& B=2 \cdot\left|V_{\mathrm{ac}}\right|^{3}\left(\frac{y_{0}}{\left|V_{\mathrm{ac}}\right|^{2}}+\frac{n_{c} \cdot Q \cdot \varepsilon \cdot t_{\mathrm{SOI}}}{k_{y_{-} \text {flex }} \cdot g_{c}}\right)
\end{aligned}
$$

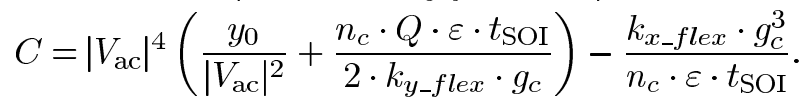

We now use (11) to express $V_{\text {dc_dyn }}$ in (7) and calculate the maximum displacement that the resonant unit can achieve before experiencing electrostatic pull-in

$$
y_{\max \_i s}=\frac{n_{c} \cdot Q \cdot \varepsilon \cdot t_{\mathrm{SOI}}}{k_{y_{-} \text {flex }} \cdot g_{\mathrm{comb}}}\left(V_{\mathrm{dc} \_d y n}+\left|V_{\mathrm{ac}}\right|\right)^{2} .
$$

For stable operation of MEMS-microlens units, this maximum displacement before side-thrust must be larger than the desired resonant amplitude.

2) Lower and Upper Limits on Resonant Frequencies: The lower limit on the resonant frequencies is related to the physical size of the rectangular unit cell. In our design, the maximum length of flexures that can be placed inside the unit cell is 1250 $\mu \mathrm{m}$, which gives rise to the lowest resonant frequency of 0.99 $\mathrm{kHz}$. However, to provide a margin of safety (because of possible variations in voltage values as well as tolerance limits in surface micromachining), it is reasonable to set the maximum length (and corresponding minimum resonant frequency) to 900 $\mu \mathrm{m}$ and $1.56 \mathrm{kHz}$, respectively.

After we have the flexure length for the lowest frequency unit, we can calculate the flexure length for the highest frequency unit based on the stationary side-thrust condition of the lowest frequency unit. The worst case that we need to consider occurs when the highest frequency unit (unit 5 in Fig. 3 ) is resonating. In this condition, we need to assure that the lowest frequency unit, which has the most pliant flexures, remains stationary and stable. Hence we first calculate the stationary side-thrust voltage for the lowest frequency unit at the stationary (rest) position. This side-thrust voltage of the lowest frequency unit is the highest voltage that can be applied to the 


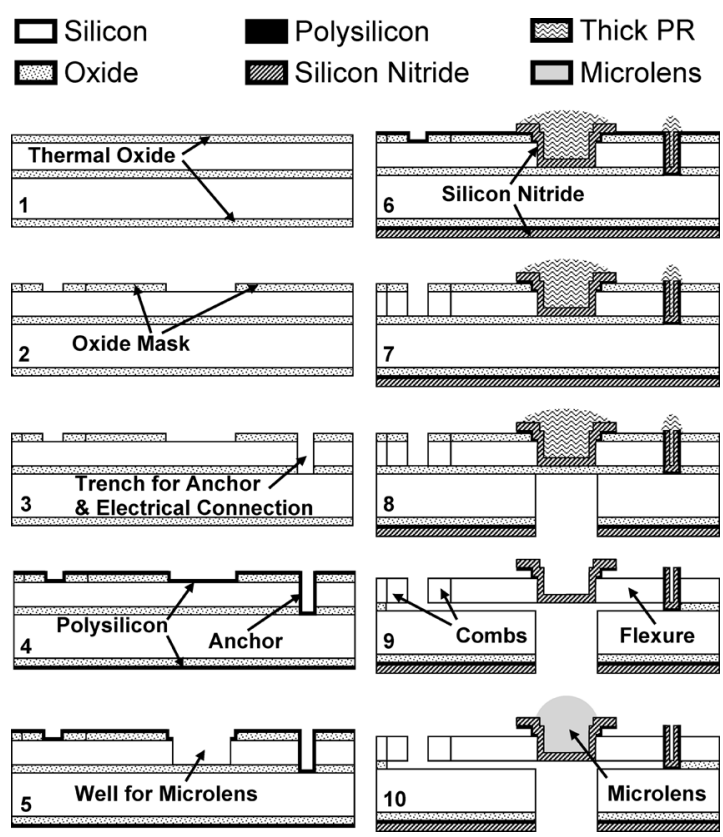

Fig. 7. Fabrication process of addressable microlens array.

1) Grow a $1-\mu \mathrm{m}$-thick thermal-silicon dioxide layer on a SOI wafer.

2) Pattern the layer to make a mask that will later define combs, flexures, supports, and lens frames.

3) Deep reactive ion etch (DRIE) trenches that will be used to form anchors and electrical connections between the device layer and the handling layer of the SOI wafers.

4) Deposit a $0.5-\mu \mathrm{m}$-thick low-pressure chemical vapor deposition (LPCVD) polysilicon layer to create electrical contacts from the device layer to the handling layer (which serves as a ground). The polysilicon layer also protects the oxide mask.

5) DRIE circular trenches in the device layer. These trenches will serve as wells for droplet microlenses later.

6) Deposit (using LPCVD) a 2- $\mu$ m-thick silicon nitride layer (tensile stress, $\sim 250 \mathrm{MPa}$ ) and pattern the layer.

7) DRIE silicon parts (combs, flexures, supports, and lens frame) using the silicon dioxide mask layer defined in step 2).

8) Open the backside of the wafers using DRIE to make clear apertures for microlenses.

9) Release the devices in concentrated HF.

10) Make microlenses using polymer-jet printing technology. The boundary of the trench defines the diameter of the lens.

common electrical interconnects in the row without causing stationary side thrust in any of the units. Then, using the stationary side-thrust voltage $\left(V_{\text {dc_st }}\right)$ of the lowest frequency unit at the stationary position, we calculate the minimum flexure length of the highest frequency unit that will allow $\pm 20-\mu \mathrm{m}$ displacement at resonance when driven at a voltage $\leq V_{\text {dc_st }}$ of the lowest frequency unit

$$
\begin{aligned}
F_{\text {side }}= & \frac{\left(n_{c} / 2\right) \cdot t_{\mathrm{SOI}} \cdot\left(y_{0}\right)}{2\left(g_{c}-x\right)^{2}}\left(V_{\mathrm{dc}}+\left|V_{\mathrm{ac}}\right|\right)^{2} \\
& -\frac{\left(n_{c} / 2\right) \cdot t_{\mathrm{SOI}} \cdot\left(y_{0}\right)}{2\left(g_{c}+x\right)^{2}}\left(V_{\mathrm{dc}}+\left|V_{\mathrm{ac}}\right|\right)^{2} \\
& +\frac{\left(n_{c} / 2\right) \cdot t_{\mathrm{SOI}} \cdot\left(y_{0}\right)}{2\left(g_{c}-x\right)^{2}}\left(V_{\mathrm{dc}}\right)^{2} \\
& -\frac{\left(n_{c} / 2\right) \cdot t_{\mathrm{SOI}} \cdot\left(y_{0}\right)}{2\left(g_{c}+x\right)^{2}}\left(V_{\mathrm{dc}}\right)^{2} .
\end{aligned}
$$

$$
\begin{aligned}
k_{x_{-} f l e x}> & {\left[\frac{\partial F_{\text {side }}}{\partial x}\right]_{x \rightarrow 0} } \\
= & \left(\frac{2 \cdot\left(n_{c} / 2\right) \cdot t_{\mathrm{SOI}}}{g_{c}^{3}}\right) \\
& \times\left[\left(y_{0}\right)\left(V_{\mathrm{dc}}+\left|V_{\mathrm{ac}}\right|\right)^{2}+\left(y_{0}\right) V_{\mathrm{dc}}^{2}\right] .
\end{aligned}
$$

Solving (9) for $V_{\mathrm{dc}}$, we obtain

$$
V_{\mathrm{dc} \_s t}=\frac{-2 \cdot\left|V_{\mathrm{ac}}\right|+\sqrt{4 \cdot\left|V_{\mathrm{ac}}\right|^{2}-8\left(\left|V_{\mathrm{ac}}\right|^{2}-D\right)}}{4}
$$

where

$$
D=\frac{k_{x_{-} \text {flex }} \cdot g_{c}^{3}}{n_{c} \cdot \varepsilon \cdot t_{\mathrm{SOI}} \cdot y_{0}} .
$$

For $l_{f}=900 \mu \mathrm{m}$ and $\left|V_{\mathrm{ac}}\right|=4 \mathrm{~V}, V_{\mathrm{dc} \_s t}$ is $44.74 \mathrm{~V}$. Using (7-1), we now calculate the maximum stiffness of the flexures $\left(k_{y \_ \text {max }}\right)$ that can still achieve $\pm 20-\mu \mathrm{m}$ resonant amplitude $\left(y_{d}\right)$ at this voltage

$$
\begin{aligned}
y_{d} & \approx \frac{n_{c} \cdot Q \cdot \varepsilon \cdot t_{\mathrm{SOI}}}{k_{y_{-} \max } \cdot g_{\mathrm{comb}}}\left(2 \cdot V_{\mathrm{dc} \_s t} \cdot\left|V_{\mathrm{ac}}\right|\right) \\
& =\frac{n_{c} \cdot \sqrt{k_{y_{-} \max } \cdot m} \cdot \varepsilon \cdot t_{\mathrm{SOI}}}{k_{y_{-} \max } \cdot g_{\mathrm{comb}} \cdot b}\left(2 \cdot V_{\mathrm{dc \_ st}} \cdot\left|V_{\mathrm{ac}}\right|\right) \\
k_{y_{-} \max } & \approx\left[\frac{n_{c} \cdot \varepsilon \cdot t_{\mathrm{SOI}} \cdot \sqrt{m}}{y_{d} \cdot g_{\mathrm{comb}} \cdot b}\left(2 \cdot V_{\mathrm{dc \_ st}} \cdot\left|V_{\mathrm{ac}}\right|\right)\right]^{2} \cdot
\end{aligned}
$$

The damping factor $b[(5)]$ increases gradually as the flexure lengthens; however, for resonant frequencies between 1-10 $\mathrm{kHz}$, the change in $b$ is typically less than $10 \%$ of its original value in our design. Hence, to simplify calculation, we assume that $b$ is fixed at $0.49 \times 10^{-5} \mathrm{~N} \cdot \mathrm{s} / \mathrm{m}$. Assuming $\left|V_{\mathrm{ac}}\right|=4 \mathrm{~V}$, we obtain $k_{y \_ \text {max }}=79.57 \mathrm{~N} / \mathrm{m}$, and the corresponding maximum resonant frequency and quality factor are $5.84 \mathrm{kHz}$ and 437.91 , respectively. Using the numerical value of $k_{y_{-} \max }$ in (3) and solving for $l_{f}$, we obtain

$$
l_{f}=\left(\frac{16 \cdot t_{\mathrm{SOI}} \cdot w_{f}^{3} \cdot E_{\mathrm{Si}}}{k_{y_{-} \max }}\right)^{1 / 3}=352 \mu \mathrm{m} .
$$

Equation (17) shows that the minimum length of the flexures allowed in a row (that can achieve $\pm 20-\mu \mathrm{m}$ displacement at resonance without causing side-thrust in low-frequency units) is $352 \mu \mathrm{m}$. This minimum length determines that the upper limit on the resonant frequencies allowed in the row [using (1)-(3)] is $5.84 \mathrm{kHz}$. As before, if we provide a safety factor (e.g., select $l_{f}=500$ instead of $352 \mu \mathrm{m}$ as the minimum flexure length), we calculate an upper limit for the resonant frequency of $4 \mathrm{kHz}$.

3) Frequency Response of the Resonant Units in a Single Row: Resonant Frequencies and Quality Factors: After choosing the design values that bracket upper and lower resonant frequencies of the MEMS-microlens units in a given row, we calculate the number of units having addressable resonant frequencies that can be placed in the row.

We predict the frequency response of the MEMS-microlens resonant structures in a row using (18) [15]

$$
y_{\mathrm{ac}}(\omega)=\frac{\left(2 \cdot V_{\mathrm{dc}} \cdot V_{\mathrm{ac}}+V_{\mathrm{ac}}^{2}\right) \cdot \varepsilon \cdot t_{\mathrm{SOI}}}{g_{\mathrm{comb}} \cdot\left[\left(k-m \cdot \omega^{2}\right)^{2}+\left(\frac{\omega \cdot \omega_{r} \cdot m}{Q}\right)^{2}\right]^{\frac{1}{2}}} .
$$




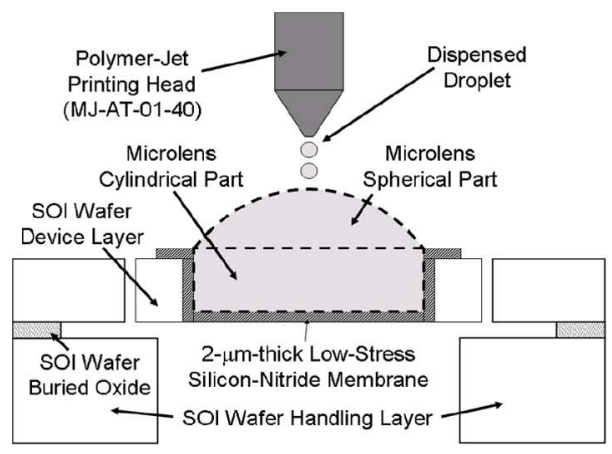

(a)

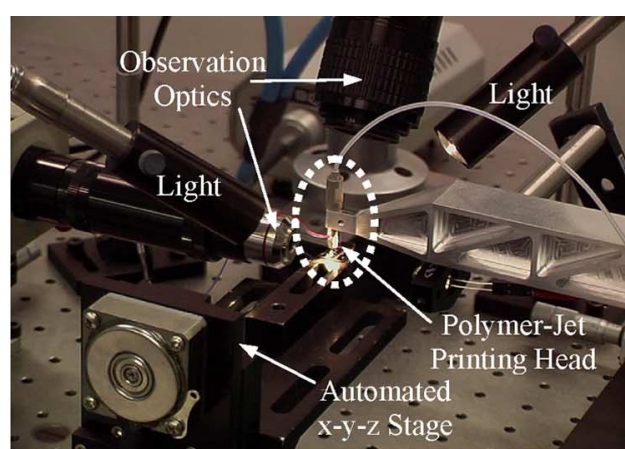

(b)

Fig. 8. Microlens fabrication process: (a) fabrication diagram and (b) fabrication setup in our laboratory.
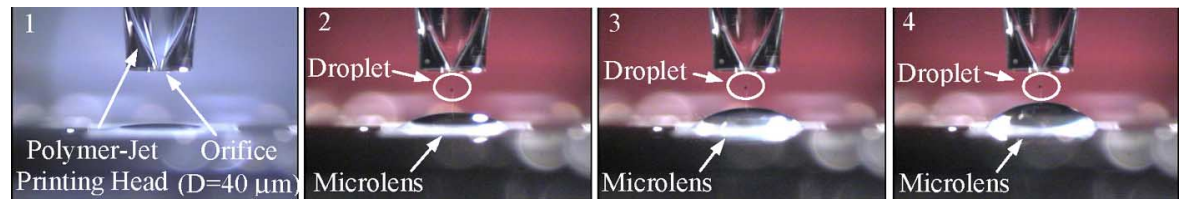

Fig. 9. Microlens fabrication process: stroboscopic observation-the focal length is controlled by varying the volume of the microlens.
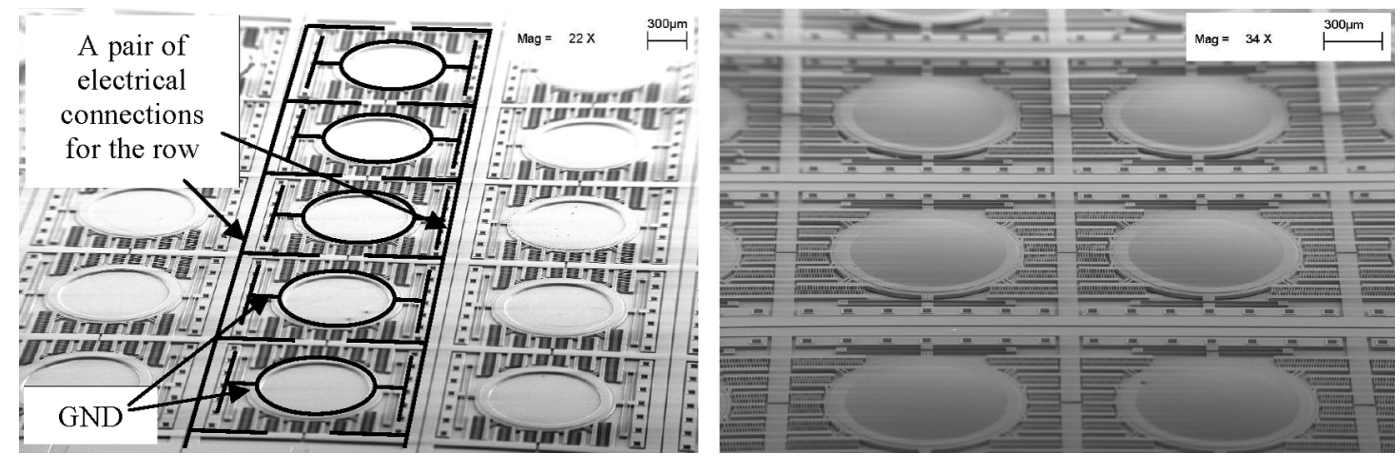

Fig. 10. SEM picture of the fabricated addressable microlens array (a) before and (b) after microlens fabrication.

Equation (18) can be used to ensure that at the resonant frequency of the unit of interest, the other units in the row do not show any significant movement.

As a design example, we fabricated a lens array in which five MEMS-microlens units (as described in Table I) were placed in a row having the dimensions indicated in Table III. Predicted resonant frequencies and mechanical quality factors for the units are also given in Table III, while in Table IV, we show predicted amplitudes for the units when they are driven at five different drive frequencies and voltages. Theory predicts that when a designated unit is at full resonance reaching amplitudes of $\pm 20 \mu \mathrm{m}$, the other four units move less than $\pm 1 \mu \mathrm{m}$, as shown in Fig. 6 . The appropriate spacing between neighboring resonant frequencies together with stability condition determines the maximum number of units that can be placed in each row for a given maximum frequency of driving voltage.

\section{FAbrication of AdDRESSAble Microlens ARRAY}

Our addressable-microlens array is fabricated in two steps: A) the MEMS-microlens carriages are built using a
Berkeley Sensor and Actuator Center (BSAC)-conventional silicon-MEMS process and $\mathrm{B}$ ) microlenses are formed on the MEMS carriages using polymer-jet printing technology developed in our laboratory [19].

\section{A. Fabrication of MEMS-Microlens Carriages}

Steps in the fabrication process of MEMS-microlens carriages are shown in Fig. 7 and described in the figure caption.

In order to make high-quality lenses using polymer-jet printing technology, it is necessary to form very flat, optically transparent diaphragms on the MEMS carriage structures. We have used LPCVD-deposited silicon nitride thin-film membranes [step 6) in Fig. 7] and found them to be excellent for this purpose. As demonstrated in previous work [20], [21], low tensile-stress ( $250 \mathrm{MPa})$ LPCVD-deposited silicon nitride membranes (5 mm squares) are virtually flat (radius of curvature $\sim 51 \mathrm{~m}$ ) and physically very robust [22]. Silicon nitride membrane also show excellent spectral transmission in the visible region (75-95\%). These excellent qualities combine to make the membranes very good choices for microlens substrates. 

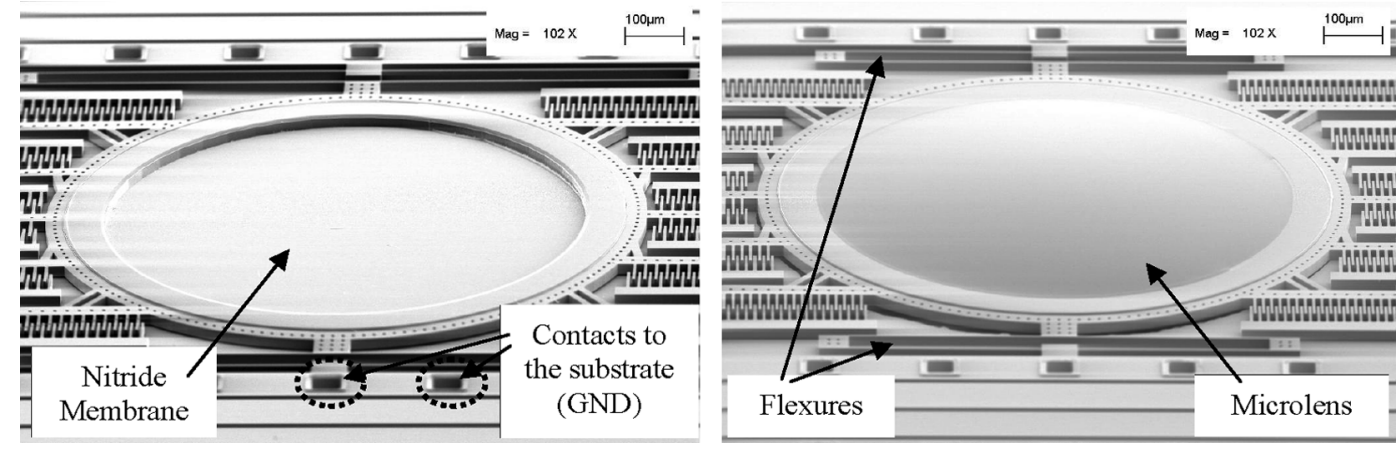

Fig. 11. SEM picture of the fabricated individual MEMS-microlens unit before and after microlens fabrication.

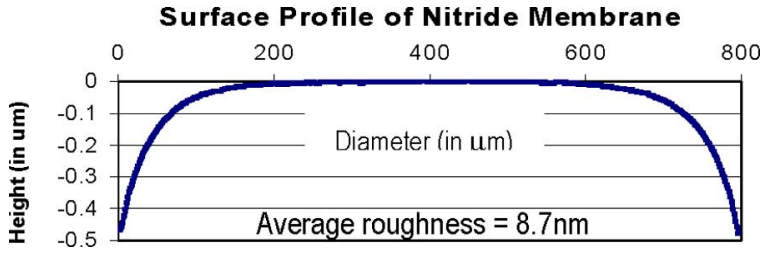

Fig. 12. WYKO measurement of low tensile-stress (250 MPa) nitride-membrane surface profile: very flat (radius of curvature $\geq 3 \mathrm{~m}$ ) within $200 \mu \mathrm{m}$ radius.

\section{B. Direct Fabrication/Integration of Microlenses on MEMS-Carriages}

Fabrication of high-quality droplet microlenses using hydrophobic effects [22] or polymer-jet printing technology [23], [24] has been reported by other researchers. In earlier research, we reported the fabrication of high-quality microlenses with excellent uniformity by combining the hydrophobic-effect method with polymer-jet printing technology [19]. To form the SH lens array, we repeated this established method depositing the polymer lenses on preformed silicon nitride substrates supported by the individual lens carriages.

The photograph on the right-hand side of Fig. 8 shows the microlens-fabrication setup in our laboratory. The microlenses are precisely formed by polymer-jet printing on $2-\mu \mathrm{m}$-thick silicon nitride layers that are uncovered by etching circular wells ( 20 $\mu \mathrm{m}$ in diameter) into the device layer of SOI wafers. The boundaries of the circular wells precisely define the lens diameters, and surface tension in the polymer creates a high-quality optical surface. The polymer-jet printing head used in our system is the Microfab MJ-AT-01-40, which operates at room temperatures. ${ }^{1}$ The MJ-AT-01-40 requires that the viscosity of the printed material not exceed $40 \mathrm{cps}$. We used Epoxy Technology's uv-curable Epo-Tek OG146, which meets this requirement at room temperature. ${ }^{2}$ In addition to its low viscosity, Epo-Tek OG146 possesses excellent optical properties: more than $95 \%$ spectral transmission after curing in the $0.4-2 \mu \mathrm{m}$ (visible to near-infrared regions) range and a refractive index of 1.51.

Optical properties such as the focal length for a microlens are adjusted by controlling the volume of deposited polymer material [19]. The total microlens volume is the sum of the spherical part and the cylindrical part, as shown in Fig. 8(a). To give

\footnotetext{
${ }^{1}$ Microfab Technologies, Inc., Plano, TX.

${ }^{2}$ Epoxy Technology, Billerica, MA.
}

an example: we fabricate a 2-mm-focal-length microlens on the MEMS-microlens carriage by depositing $2.98 \times 10^{-11} \mathrm{~m}^{3}$ (or $29.8 \mathrm{nl}$ ) of the microlens material. Since the polymer-jet printing head generates a droplet of $0.025 \mathrm{nl}$, we need to deposit 1192 drops to fabricate a microlens having the required properties on the MEMS carriage.

\section{EXPERIMENTAL RESULTS AND DISCUSSION}

Examples of our $5 \times 5$ addressable-lens arrays, fabricated using SOI wafers, are pictured in the scanning electron microscope (SEM) photographs shown in Figs. 10 and 11. Each addressable unit $\left(1.5 \mathrm{~mm}^{2}\right)$ contains one $800-\mu \mathrm{m}$-diameter microlens with lens-support carriage and actuators.

\section{A. Microlens}

Using WYKO-NT3300, we measured the surface profiles of the low-stress $(\sim 250 \mathrm{MPa})$ tensile-silicon nitride-membranes and microlenses $(\mathrm{EFL}=5.5 \mathrm{~mm}$ ) (Figs. 12 and 13). Within a $200-\mu \mathrm{m}$ radius, the membranes are virtually flat (radius-of-curvature $\geq 3 \mathrm{~m}$ ) (Fig. 12). Near the edge, the membrane profiles deviate slightly from ideal flat surfaces, but the maximum deviation across its $800 \mu \mathrm{m}$ diameter is still lower than $0.5 \mu \mathrm{m}$. The average surface roughness is measured at $8.7 \mathrm{~nm}$.

Using our polymer-jet printing technique in circular wells, we have been able to produce microlenses with effective focal lengths (EFLs) ranging from 1.94 to $7.48 \mathrm{~mm}$ as adjusted by controlling the deposited polymer volumes forming the microlenses. Fig. 12 shows the surface profile of a microlens $(\mathrm{f}=5.5 \mathrm{~mm})$ and its deviation from that of an ideal circle. The microlens surfaces closely approximate a circle having radius $2.2 \mathrm{~mm}$.

For our addressable-microlens array, microlenses having a designed focal length of $2.0 \mathrm{~mm}$ were fabricated. The $25 \mathrm{fab}-$ ricated microlenses have an average EFL of $2.09 \mathrm{~mm}$, and the peak-to-peak variation in EFL is $\leq 7 \%$.

\section{B. Mechanical Performance}

The measured mechanical resonant frequencies of the MEMS-microlens units 1 through 5 (microlens EFL $=2.09 \mathrm{~mm}$ ) are $1.31,1.58,1.98,2.48$, and $3.49 \mathrm{kHz}$, respectively. The corresponding mechanical Q-factors (microlens $\mathrm{EFL}=2.09 \mathrm{~mm}$ ) are 65.4, 105.1, 142.1, 174.8, and 205.2. Across the same chip, the maximum variation in resonant frequencies of five identical units is less than 5\%. All units achieve 

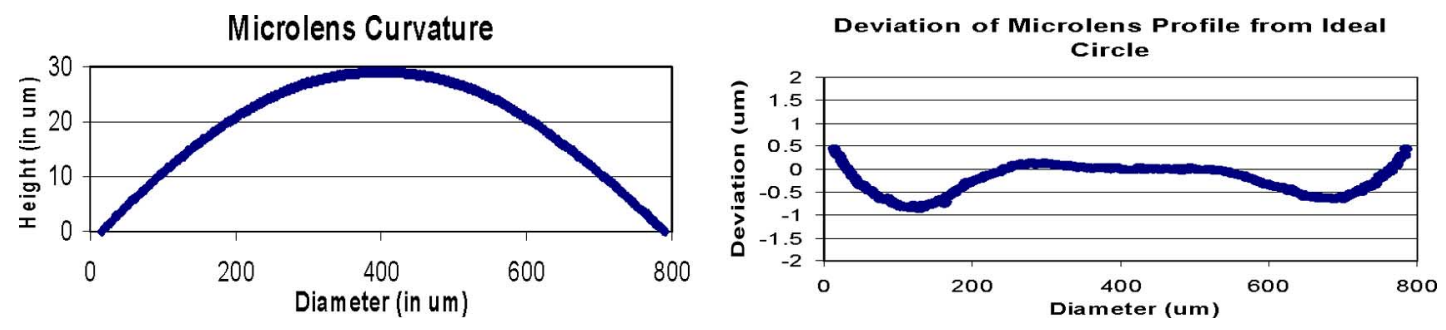

Fig. 13. The microlens $(\mathrm{f}=5.5 \mathrm{~mm}$ ) profile follows closely with an ideal circle (radius $=2.2 \mathrm{~mm}$ ) within $200 \mu$ m radius. Its deviation near the edge may be influenced by the profile of the nitride membrane underneath it.
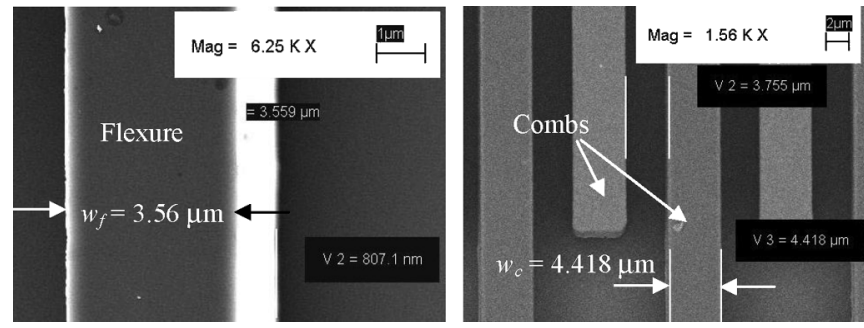

Fig. 14. Overetched combs and flexures during the DRIE process.

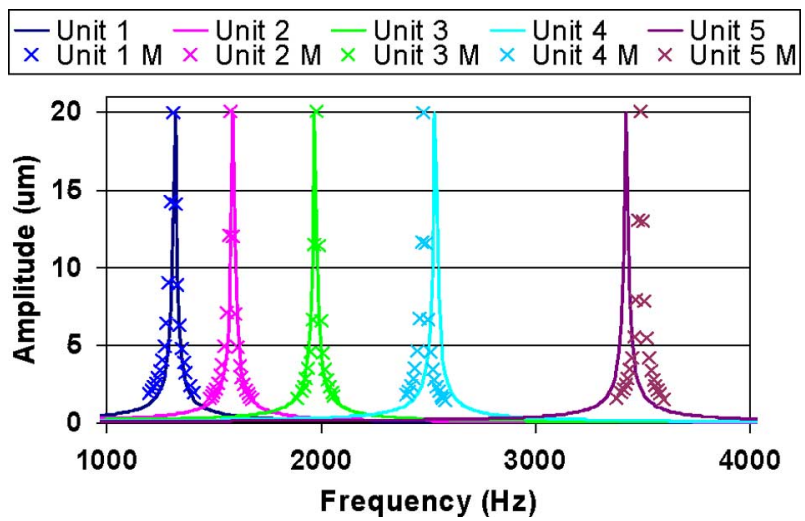

Fig. 15. Comparison between the theoretical model and experimentally measured data: Units 1-5 indicate the theoretical prediction while Units $1 \mathrm{M}-5 \mathrm{M}$ present the experimental measurements.

TABLE V

Processing VARIaTION ON STRUCTURE DimEnSIONS

\begin{tabular}{|c|c|c|}
\hline & Design & Measured \\
\hline $\boldsymbol{w}_{f}(\boldsymbol{\mu m})$ & 4 & 3.57 \\
\hline $\boldsymbol{w}_{c}(\boldsymbol{\mu m})$ & 5 & 4.418 \\
\hline
\end{tabular}

$40 \mu \mathrm{m}$ resonant excursions $( \pm 20 \mu \mathrm{m})$ when applying actuation voltages equal to or lower than $4 \mathrm{~V}_{\text {ac_peak-to-peak }}+44 \mathrm{~V}_{\mathrm{dc}}$ are applied. There was no noticeable mechanical crosstalk observed under study with an optical microscope.

The experimentally measured resonant frequencies deviate from their design values by as much as $17 \%$ due mainly to imprecision in control of the DRIE process as revealed by SEM measurements and shown in Fig. 14 and Table V.

Using the dimensions given in Table $\mathrm{V}$, our calculations for the resonant frequencies nearly match the measured values as indicated in Table VI.

Precisely estimating the quality factors using theoretical models can be quite challenging [25]. As other researchers have
TABLE VI

EXPERIMENTALLY MEASURED AND THEORETICALLY PREDICTED RESONANT FREQUENCIES AND QUALITY FACTORS

\begin{tabular}{|c|c|c|c|c|c|}
\hline & Unit1 & Unit2 & Unit3 & Unit4 & Unit5 \\
\hline $\boldsymbol{l}_{\boldsymbol{f}}(\boldsymbol{\mu \mathrm { m }})$ & $\mathbf{9 0 0}$ & $\mathbf{8 0 0}$ & $\mathbf{7 0 0}$ & $\mathbf{6 0 0}$ & $\mathbf{5 0 0}$ \\
\hline $\boldsymbol{f}_{\boldsymbol{r}}(\boldsymbol{k H z})$ calculated & 1.32 & 1.59 & 1.97 & 2.53 & 3.42 \\
\hline $\boldsymbol{f}_{\boldsymbol{r}}(\boldsymbol{k H z})$ measured & 1.31 & 1.58 & 1.98 & 2.48 & 3.49 \\
\hline $\boldsymbol{Q}$ calculated & 99.0 & 119.1 & 147.1 & 188.2 & 252.6 \\
\hline $\boldsymbol{Q}$ measured & 65.4 & 105.1 & 142.1 & 174.8 & 205.2 \\
\hline
\end{tabular}

found previously, (12) underestimates the damping factor and, as a result, the quality factors are overestimated [18], [25].

Individual frequency-addressing of MEMS-microlens resonant units in a row is demonstrated in Figs. 16 and 17. In all five cases, the targeted unit achieves highly distinguishable resonance $( \pm 20 \mu \mathrm{m})$ while the others in the row are effectively still.

\section{CONCLUSION}

We have demonstrated a (5 by 5) addressable array of high-quality microlenses that can be applied to a Shack-Hartmann sensor in a microoptical system to improve its dynamic range. Specific lenses in the array can be addressed using our new design in which the mechanical resonant frequencies of individual lens-support carriages are varied. The measured mechanical resonant frequencies of the MEMS-microlens units 1 through 5 with microlenses $(\mathrm{EFL}=2.09 \mathrm{~mm}$ ) range from 1.31 up to $3.49 \mathrm{kHz}$, and the corresponding Q-factors were between 65.4 and 205.2. All units achieve $40 \mu \mathrm{m}$ resonant excursions $( \pm 20 \mu \mathrm{m})$ when applying actuation voltages $\left(\left|\mathrm{V}_{\mathrm{ac}}+\mathrm{V}_{\mathrm{dc}}\right|\right)$ lower than $50 \mathrm{~V}$. Optically observed mechanical crosstalk between different units is negligible. Use of the frequency-selection scheme to address individual MEMS structures in an array is not limited to the optical application demonstrated in this project. This scheme clearly has value for other MEMS applications such as print-head activation in multicolor printers or activated drug dispensing, to cite only two examples. In each of these cases, an important advantage of the selection technique is that it does away with the need for multiple input-addressing leads or for the use of sophisticated electronics.

\section{ACKNOWLEDGMENT}

The authors would like to thank J. Seeger for technical discussions on resonator design, Dr. H. Kim of Hewlett Packard Lab (Palo Alto, CA) for helpful discussion on nitride-membrane fabrication, Dr. B. Guildman for helpful discussions on 


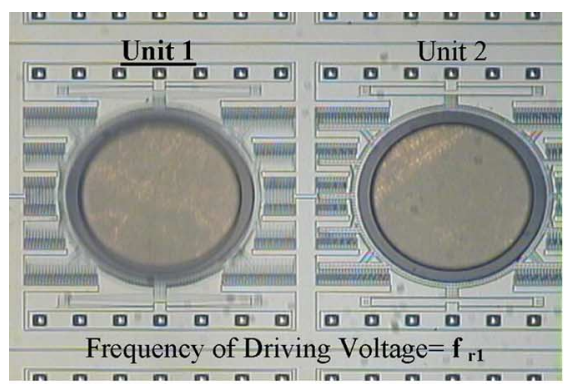

(a)

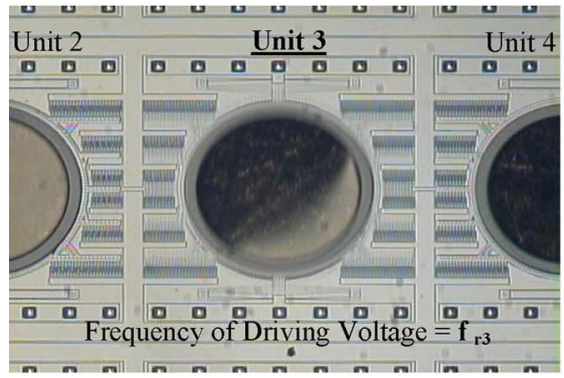

(c)

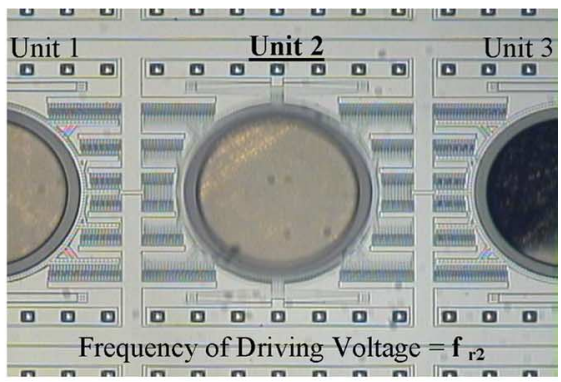

(b)

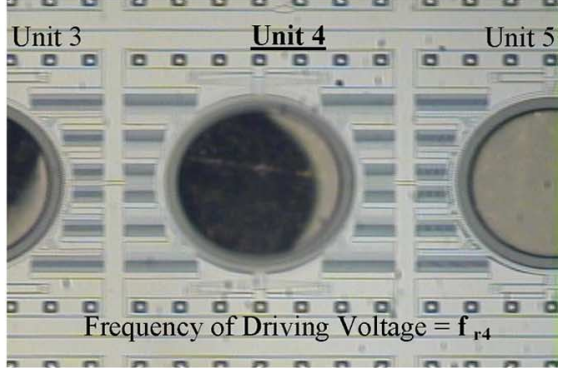

(d)

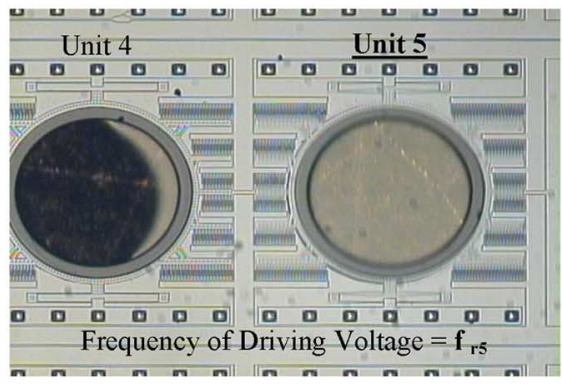

(e)

Fig. 16. To be aware of the motions, note the flexures of the each unit shown in the images: (a) Unit 1 is at resonance. Unit 2 is still. (b) Unit 2 is at resonance. Units 1 and 3 are still. (c) Unit 3 is at resonance. Units 2 and 4 are still. (d) Unit 4 is at resonance. Units 3 and 5 are still. (e) Unit 5 is at resonance. Unit 4 is stationary. (Nonuniform metallic texture seen on the nitride membrane is a reflection of the surface of the copper wafer-chuck. The dark shadows on the membranes for Units 3 and 4 are due to the objective lens of the optical microscope.)

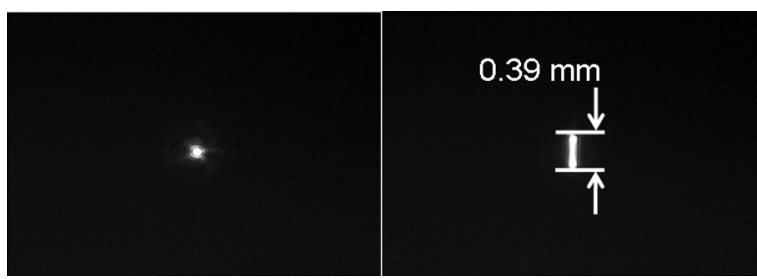

Fig. 17. Demonstration of focal-point identification: As the microlens resonates, its focal point generates a scanning line, and the focal point and its associated microlens can thereby be identified. The focal length $f$ of the microlens is $2.09 \mathrm{~mm}$, and the CCD imager is placed $9.84 \mathrm{f}$ from the lens. The focal point travels $\sim 40 \mu \mathrm{m} \times 9.84 \mathrm{f}$ or $\sim 0.39 \mathrm{~mm}$ on the CCD imager.

deep-trench photolithography, Prof. J. Bokor for helpful discussion on optics, and J. Black for wire-bonding. The authors also thank Prof. K. S. J. Pister and his graduate researcher S. Bergbreiter at the University of California (UC), Berkeley, for providing SOI wafers and B. Hamilton of UC Berkeley Microlab for invaluable advice on polymer-jet printer setup.

\section{REFERENCES}

[1] E. Viard, M. Le Louarn, and N. Hubin, "Adaptive optics with four laser guide stars: Correction of the cone effect in large telescopes," Appl. Opt., vol. 41, no. 1, pp. 11-20, Jan. 2002.

[2] L. N. Thibos and X. Hong, "Clinical applications of the Shack-Hartmann aberrometer," Opt. Vision Sci., vol. 76, no. 12, pp. 817-825, Dec. 1999.

[3] K. Munson, X. Hong, and L. N. Thibos, "Use of a Shack-Hartmann aberrometer to assess the optical outcome of corneal transplantation in a keratoconic eye," Opt. Vision Sci., vol. 78, no. 12, pp. 866-871, Dec. 2001.

[4] L. Llorente, L. Diaz-Santana, D. Lara-Saucedo, and S. Marcos, "Aberrations of the human eye in visible and near infrared illumination," Opt. Vision Sci., vol. 80, no. 1, pp. 26-35, Jan. 2003.

[5] X. Cheng, N. L. Himebaugh, P. S. Kollbaum, L. N. Thibos, and A. Bradley, "Validation of a clinical Shack-Hartmann aberrometer," Opt. Vision Sci., vol. 80, no. 8, pp. 587-595, Aug. 2003.

[6] C. E. Joslin, S. M. Wu, T. T. McMahon, and M. Shahidi, "Higher-order wavefront aberrations in corneal refractive therapy," Opt. Vision Sci., vol. 80, no. 12, pp. 805-811, Dec. 2003.

[7] R. V. Shack and B. C. Platt, "Production and use of lenticular Hartmann screen," in Programme 1971 Spring Meeting Opt. Soc. Amer., Washington, DC, 1971.

[8] J. Pfund, N. Lindlein, and J. Schwider, "Dynamic range expansion of a Shack-Hartmann sensor by use of a modified unwrapping algorithm," Opt. Lett., vol. 23, no. 13, pp. 995-997, Jul. 1998. 
[9] N. Lindlein, J. Pfund, and J. Schwider, "Expansion of the dynamic range of a Shack-Hartmann sensor by using astigmatic microlenses," Opt. Eng., vol. 39, no. 8, pp. 2220-2225, Aug. 2000.

[10] N. Lindlein, J. Pfund, and J. Schwider, "Algorithm for expanding the dynamic range of a Shack-Hartmann sensor by using a spatial light modulator array," Opt. Eng., vol. 40, no. 5, pp. 837-840, May 2001.

[11] S. A. Klein, "Problems with wavefront aberrations applied to refractive surgery: Developing standards," in Proc. SPIE Ophth. Technol. XI, Jan. 2001, vol. 4245, pp. 47-56.

[12] A. Tuantranont, V. M. Bright, J. Zhang, W. Zhang, J. A. Neff, and Y. C. Lee, "Optical beam steering using MEMS-controllable microlens array," Sens. Actuators A Phys., vol. A91, no. 3, pp. 363-372, Jul. 2001.

[13] H. Toshiyoshi, G.-D. J. Su, J. LaCosse, and M. C. Wu, "A surface micromachined optical scanner array using photoresist lenses fabricated by a thermal reflow process," J. Lightwave Technol., vol. 21, pp. 1700-1708, Jul. 2003.

[14] R. Legtenberg, A. W. Groeneveld, and M. Elwenspoek, "Comb-drive actuators for large displacements," J. Micromech. Microeng., vol. 6, no. 3, pp. 320-329, Sep. 1996.

[15] G. T. A. Kovacs, Micromachined Transducers: Sourcebook. New York: McGraw-Hill, ch. 3.

[16] W. C. Tang, T.-C. H. Nguyen, and R. T. Howe, "Laterally driven polysilicon resonant microstructures," Sens. Actuators, vol. 20, pp. 25-32, 1989.

[17] A. C. Ugural, Mechanics of Materials. New York: McGraw-Hill, 1990 , ch. 9.

[18] X. Zhang and W. C. Tang, "Viscous air damping in laterally driven microresonators," in Tech. Dig. IEEE Micro Electro Mech. Syst. Workshop, Jan. 1994, pp. 199-204.

[19] H. Choo and R. S. Muller, "Optical properties of microlenses fabricated using hydrophobic effects and polymer-jet-printing technology," in Proc. 2003 IEEE/LEOS Int. Conf. Opt. MEMS Applicat., Waikoloa, HI, Aug. 18-21, 2003, pp. 169-170.

[20] D. R. Ciarlo, "Silicon nitride thin windows for biomedical microdevices," Biomed. Microdev., vol. 4, no. 1, pp. 63-68, Mar. 2002.

[21] K. Gupta, H. Choo, H. Kim, and R. S. Muller, "Micromachined polarization beam splitters for the visible spectrum," in Proc. 2003 IEEE/ LEOS Int. Conf. Opt. MEMS Applicat., Waikoloa, HI, Aug. 18-21, 2003, pp. 171-172.

[22] D. M. Hartmann, O. Kibar, and S. C. Esener, "Optimization and theoretical modeling of polymer microlens arrays fabricated with the hydrophobic effect," Appl. Opt., vol. 40, no. 16, pp. 2736-2746, Jun. 1, 2001.

[23] D. L. MacFarlane, V. Narayan, J. A. Tatum, W. R. Cox, T. Chen, and D. J. Hayes, "Microjet fabrication of microlens arrays," IEEE Photon. Technol. Lett., vol. 6, pp. 1112-1114, Sep. 1994.

[24] C. Ting, W. R. Cox, D. Lenhard, and D. J. Hayes, "Microjet printing of high-precision microlens array for packaging of fiber optic components," in Proc. SPIE Int. Soc. Opt. Eng., 2002, vol. 4652, pp. 136-141.

[25] Y.-H. Cho, A. P. Pisano, and R. T. Howe, "Viscous damping model for laterally oscillating microstructures," J. Microelectromech. Syst., vol. 3, no. 2, pp. 81-87, Jun. 1994.

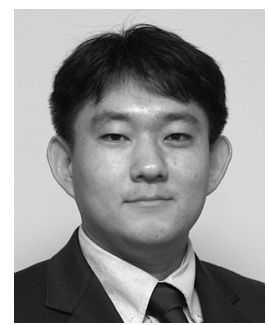

Hyuck Choo received the B.S. and M.Eng. degrees in 1996 and 1997, respectively, in electrical engineering from Cornell University, Ithaca, NY. He is currently pursuing the $\mathrm{Ph} . \mathrm{D}$. degree in electrical engineering and computer sciences at the University of California, Berkeley (UC Berkeley), under Prof. R. S. Muller's supervision.

Before enrolling at UC Berkeley, he was with Kionix, Inc., Ithaca, NY, as a MEMS test engineer. $\mathrm{He}$ has focused his research on microlens and microscanner systems, particularly on applications to ocular-refractive surgery, biomedical-imaging systems, high-definition displays, and next-generation wavefront sensors. His most recent projects are directed toward the development of fast MEMS-based phase-shifting interferometers and their applications to the measurement of transient optical and biological phenomena.

Mr. Choo won the UC Berkeley EECS Lim Prize in 2001 (Best Performance on the Pre-Doctoral Exam) and is the two-time winner of the Berkeley Sensor and Actuator Center presentation award.

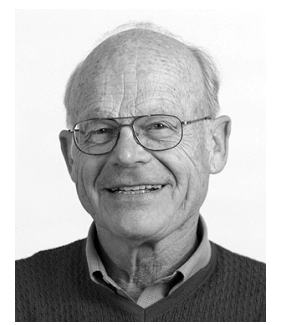

Richard S. Muller (S'57-M'58-SM'70-F'88LF'97) received the mechanical engineer's degree from Stevens Institute of Technology, Hoboken, NJ, the M.S. (electrical engineering) and Ph.D. degree (electrical engineering and physics) degrees in 1962 from the California Institute of Technology, Pasadena.

After employment as a Member of the Technical Staff at Hughes Aircraft Company, he joined the faculty at the University of California, Berkeley, where he concentrated his research on the physics of integrated-circuit devices. Together with Dr. T. I. Kamins of Hewlett-Packard Company, he first published, Device Electronics for Integrated Circuits in 1977. A third edition of this book (which has been translated into five languages) was published in 2003. In the late 1970s, he began research in the area now known as MEMS and, together with R. M. White, he founded the Berkeley Sensor and Actuator Center in 1986. His present research focus is on optical MEMS.

Dr. Muller wrote the proposal to establish the IEEE/ASME JOURNAL of Microelectromechanical Systems (JMEMS) and is now the Editor-in-Chief of this journal. A member of the U.S. National Academy of Engineering, he received a career MEMS Award at TRANSDUCERS '97 as well as the IEEE Brunetti Award (1998 with R. T. Howe), a Fulbright Professorship, and a von Humboldt Research Award at TU Berlin in 1994. His other awards include the Berkeley Citation and the Renaissance Award from Stevens Institute of Technology, where he served as Trustee 1996-2005. He has been a member of the National Materials Advisory Board and served on several National Research Council study panels as well as chairing a 1997 panel for which he acted as editor of a widely distributed report on the promises and challenges of MEMS. His present research focus is on optical MEMS. He is a Member of the American Society of Mechanical Engineers (ASME). 